\title{
Research Tools for Understanding Sports Consumers
}

\author{
Peng Cui ${ }^{1, a}$ \\ ${ }^{1}$ School of Business management, Tonghua Normal University, China \\ adbcy9999@163.com
}

Keywords: consumers; marketing research; research tools; sports marketing; research process Abstract. This paper is focus on discuss the importance of marketing research to sports marketers. Explain the fundamental process for conducting sports marketing research. Identify the various research design types. Describe the process for questionnaire development. Understand how to prepare an effective research report.

\section{Introduction}

Marketing research is a fundamental tool for understanding and ultimately satisfying customers' need. As described before one way of demonstrating a marketing orientation to gather information used for decision making. Another way of establishing a marketing orientation is to disseminate information and share the marketing information with those responsible for making decisions. Marketing research is viewed as an essential element in marketing-oriented organizations.

The information gathered through marketing research can be as basic as where consumers live, how much money they make, and how old they are. Research also provides information for decision makers in identifying marketing opportunities and threats, segmenting markets, choosing and understanding the characteristics of target markets, evaluating the current market positioning, and marketing mix decisions.

Three key issues emerge from this definition. First, marketing research must be systematic in its approach. Systematic research is both well organized and unbiased. The well-organized nature of good research is dependent on adherence to the marketing research process, which is discussed later in this paper.

Second, the marketing research process involves much more than collecting data and then reporting it back to decision makers the challenge of research lies in taking the data collected, analyzing it, and then making sense of the data. Marketing researchers who can collect data, dump it in the computer, and spit out reports are a dime a dozen. The most valuable marketing researcher is the person who has the ability to examine the data and then make recommendations about how information should be used (or not used) in the strategic marketing process.

Third, the information of marketing research is found in its ability to allow managers to make informed decisions [1].

Finally, the definition states that marketing research is useful throughout the entire strategic sports marketing process. Traditionally, the focus of marketing research has been on how the information can be used in better understanding consumers during the phase of the strategic sports marketing process. It is also important to realize that marketing research is relevant at the implementation and control phases of the strategic marketing process.

\section{The marketing research process}

As previously mentioned, marketing research is conducted using a systematic process, or the series of interrelated steps shown in Figure 1, Before discussing each step in the research process in greater detail, two points should be kept in mind. First, the basic framework or process for conducting marketing research does not change, although every marketing research problem will. Each of these research questions is different. However, the basic marketing research process used to address each question is the same. 
Second, you should understand that the steps of the research process are interdependent. In other words, defining the problem in a certain way will affect the choice of research design. Likewise, selecting a certain type of research design will influence the selection of data collection tools. Let us now examine each of the steps in the research process.

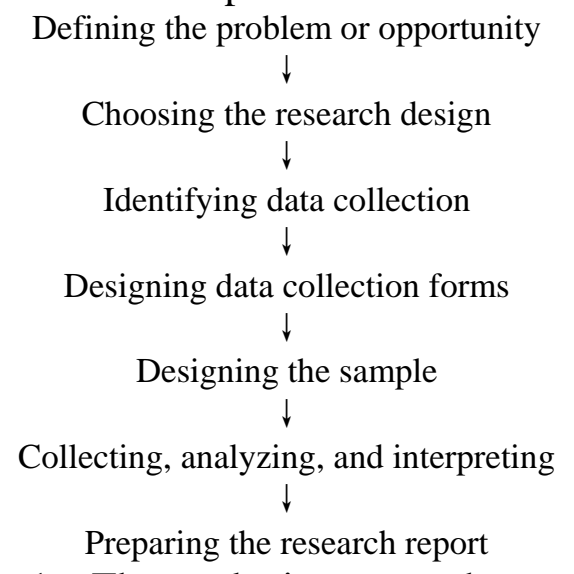

Fig. 1 - The marketing research process

\section{Defining the problem or opportunity}

The first and most important step of the marketing research process for sports marketers is to define the problem or opportunity. Problem definition requires the researcher to specify what information is needed to assist in either solving problems or identifying opportunities by developing a research problem statement. If the research addresses the correct problem or opportunity and seeks to properly define the problem or opportunity, then the project could be successful. However, the data collected may be useless if it is not the information needed by the sports marketing manager [2].

\section{Choosing the research design type}

Once the research is certain that problem is correctly defined, the research design type [3] is considered. The research design is the framework for a study that collects and analyzes data. Although every study is unique and requires a slightly different plan to reach the desired goals and objectives, three research design types have emerged. These design types are called exploratory ,descriptive, and causal designs. Whatever research design or designs are ultimately chosen, it is important to remember the crucial principle in research is that the design of the research should stem from the problem.

Exploratory designs are useful when research problems are not well defined. In addition to describing the characteristics of a targeted group, descriptive designs show the extent to which two variables differ or correlate. For example, a researcher may want to examine the relationship between game attendance and merchandising sales. If a positive relationship was found between age and likelihood of attending games in the future, then the older you get, the more likely you would be to attend future Rive Rat games. That is, as the age of the fan increases, the likelihood of going to future games also increases (see Fig. 2a). However, a negative relationship means that as age increases, the likelihood of going to games decreases (see Fig. 2b). Knowing the shape of this relationship will help the River Rat's marketers make decisions on who to target and how to develop the appropriate marketing mix for this group. What do you think the relationship between age and attendance would look like? 

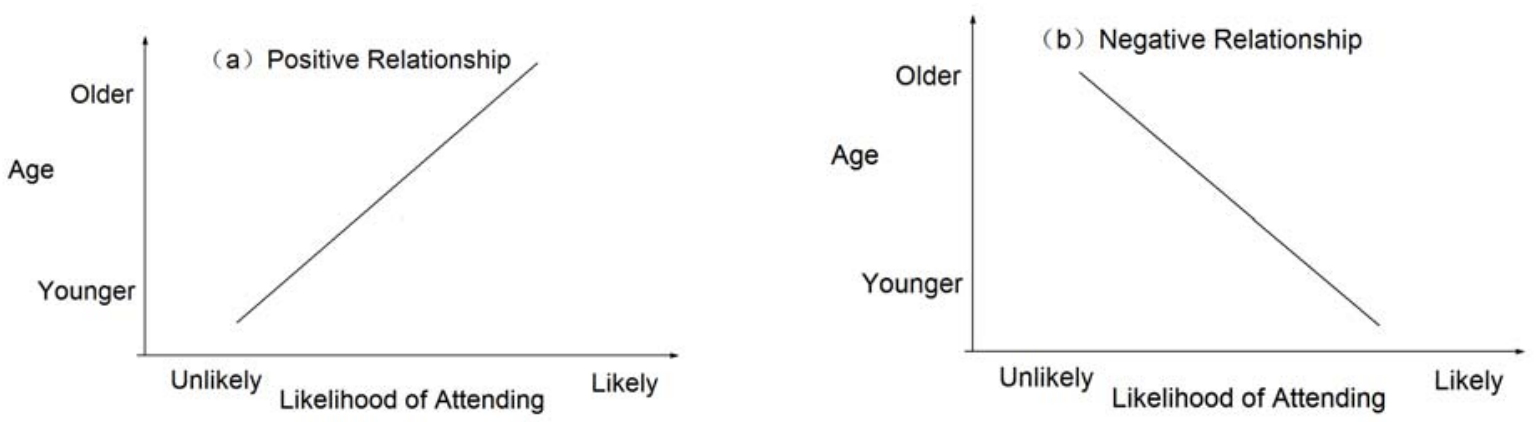

Fig. 2 - Descriptive Research Designs

Using a descriptive design, we can explore the relationship between two variables, such as age and likelihood of attending games in the future. However, what this does not tells us is that age causes the likelihood of attending to either increase or decrease. This can only be determined through a causal design [4].

Causal designs are useful when problems are very clearly defined. More specifically, causal designs examine whether changing the level of one variable causes the level of another variable to change. This is more commonly called a cause-and-effect relationship.

To show cause-and-effect relationships, three criteria must be satisfied. The first criterion for causality is that the occurrence of the causal event must precede or be simultaneous to the effect it is producing. The second criterion for causality involves the extent to which the cause and the effect vary together. This is called concomitant variation. If advertising expenditures are increased, then season ticket sales should also increase at the same rate of change. Likewise, when advertising spending is decreased, season ticket sales should also decline. Keep in mind, however. That concomitant variation does not prove a cause-and-effect relationship, but is a necessary condition for it. A third criterion used to show causal relationships requires the elimination of other causal factors. This means that another variable or variables may produce changes in the independent variable. This possibility is called a spurious association or spurious correlation. In the dynamic sports marketing environment, it could be difficult to isolate and eliminate all possible causal factors. For instance, an increase in attendance may be due to the success of the team, ticket prices, and addition of other promotions rather than increased advertising. A researcher must attempt to eliminate these other potential factors, hold them constant, or adjust the results to remove the effects of any other factors.

\section{Identifying data collection techniques}

As with the previous steps in the research process, decisions regarding data collection techniques are very much a function of problem definition and research design type. If the research problem is loosely defined and requires an exploratory research design, then there are more alternatives for collecting that information. Data collection techniques can be broadly categorized as secondary or primary. Secondary data refer to data refer to data that have already been collected but are still related to the research question. This data may come from within the sports organization or from outside the organization. One of the most comprehensive sources of secondary data that is readily available via the internet is census information. Here are some of the census documents that may be of interest: census of population, census of retail trade, census of service industries, and census of manufacturing industries.

\section{Designing data collection instruments and data analysis}

Once the data collection method has been chosen, the next step in the marketing research process is designing the data collection instrument. Data collection instruments are required for nearly all types of data collection methods. Guides are necessary for depth interviews and focus groups. Data collection forms are needed for projective techniques. Even experiments require data collection instruments. After the data have been collected from the population of interest, data analysis takes place. Before any analytical work occurs, the data must be carefully scrutinized to ensure its quality. 
We call this the editing process. During this process, the data is examined for impossible responses, missing responses, or any other abnormalities that would render the data useless.

Once the quality of data is ensured, coding begins. Coding refers to assigning numerical values or codes to represent a specific response to a specific question [5].

\section{Preparing a final report}

The last step in the marketing research process is preparing a final report [6-10]. Typically, the report is intended for top management of the sports organization, who can either put the research findings into action or shelve the project. Unfortunately, the greatest research project in the world will be viewed as a failure if the results are not clearly communicated to the target audience.

\section{Summary}

This paper focuses on the tools used to gather information to make intelligent decisions, throughout the strategic sports marketing process. More specifically, the paper describes the marketing research process in detail. Marketing research is defined as the systematic process of collecting, analyzing, and reporting information to enhance decision making throughout the strategic sports marketing process. The marketing research process consists of seven interrelated steps. These steps include defining the problem; choosing the research design type; identifying data collection methods; designing data collection forms; designing the sample; collecting, analyzing, and interpreting data; and preparing the research report.

\section{Acknowledgements}

The work was supported by Research Foundation of Education Bureau of Jilin Province (Grant No.2014388)

\section{References}

[1]Horne, J, Sport in consumer culture, 2006.

[2]Gilbert Churchill, Basic Marketing Research, 3rd ed. Dryden Press, 1996.

[3]Now That's a Name That Can Hurt You, The Seattle times, August 25, 2000.

[4]Cleveland Indians Look to Long-Term Viability through Market Research, Akron Beacon Journal, April 16, 1999.

[5]Graig Mertz, Pro Lacrosse League to Test Local Support, The Columbus Dispatch, July 7, 2000, $5 \mathrm{D}$.

[6]Barry Smart, Not playing around: global capitalism, modern sport and consumer culture, Global Networks, Vol. 7, Issue 2, 2007, 113-134.

[7]Sarah Auvergne, Sport tourism consumer experiences: a comprehensive model, Journal of Sport \& Tourism, Vol. 9, Issue 2, 2004, 127-140.

[8]Funk, D. C.; Mahony, D. F.; Ridinger, L. L.,Characterizing consumer motivation as individual difference factors: augmenting the Sport Interest Inventory (SII) to explain level of spectator support, Sport Marketing Quarterly, Vol. 11 No. 1, 1999, 33-43.

[9]Daniel C. Funk, Jeffrey D. James, Consumer Loyalty: The Meaning of Attachment in the Development of Sport Team Allegiance, Journal of Sport Management, 20, 2006, 189-217.

[10]James, J. D.; Ross, S. D., Comparing sport consumer motivations across multiple sports, Sport Marketing Quarterly, Vol. 13 No. 1, 2004, 17-25. 